Check for updates

Cite this: RSC Adv., 2018, 8, 22577

Received 18th February 2018

Accepted 17th May 2018

DOI: $10.1039 / c 8 r a 01324 a$

rsc.li/rsc-advances

\section{Preparation and properties of CA/ATP-g-CDs gel fibers for simultaneous detection and adsorption of methylene blue $\uparrow$}

\author{
Fengying Dai, Mengjuan Wen, Jinna Wang, Wenzhe Jiang, Xuetao Tian, Yao Dong, \\ Yiping Zhao (D) * and Li Chen (D) *
}

To detect and adsorb methylene blue (MB) from wastewater simultaneously, a solid fluorescent and absorbent material was designed by immobilizing attapulgite (ATP) on calcium alginate (CA) and reacting with carbon dots (CDs) which were modified by the activation of $\gamma$-(2,3-epoxypropoxy) propyltrimethoxysilane ( $\mathrm{KH}-560)$, then the CA/ATP- $g$-CDs gel fibers were prepared. The problem of CDs easily falling out of materials was solved. The structures of the gel fibers were characterized by field emission scanning electron microscopy (FE-SEM), specific surface area (BET) and X-ray photoelectron spectroscopy (XPS). The thermal properties were analyzed by thermogravimetry (TG). The adsorption capacity was measured and the effect of initial pH was investigated. The results showed that ATP was successfully reacted with $\mathrm{CA}$ and the adsorption capacity was enhanced with the increase of the $\mathrm{pH}$ value. CA/ATP-g-CDs gel fibers were favorable materials to detect and adsorb MB simultaneously, and MB could be adsorbed by gel fibers and also the fluorescence of CA/ATP-g-CDs was weakened. At low concentrations of $\mathrm{MB}\left(1 \mu \mathrm{g} \mathrm{L}^{-1}\right)$, the removal efficiency could even be as high as $100 \%$.

\section{Introduction}

Attapulgite (ATP) is a kind of natural inorganic mineral composed of hydrated magnesium aluminum silicate. The specific surface area of ATP is quite large because the microstructure of ATP is rod-like or fibrous in morphology. As an inexpensive and abundant nanomaterial, ATP has attracted great attention as an adsorbent to adsorb dye molecules ${ }^{\mathbf{1 , 2}}$ metal ions ${ }^{3,4}$ and other pollutants from aqueous media, ${ }^{5}$ which is attributed to the large specific surface area, special porous structure, strong cation exchange capacity and abundant active hydroxyl groups on the surface. ${ }^{6,7}$ However, the dispersibility of ATP in water and its nano-sized structure make it difficult to remove from water by using conventional separation methods. Therefore, secondary pollution caused by the treatment of waste water becomes one of the difficult problems that restricts the wide application of ATP in water treatment. To overcome this drawback, it is effective to prepare composites by immobilizing ATP into some suitable matrix materials.

Alginate with a linear chain structure is composed of $\alpha-1,4-\mathrm{L}^{-}$ glucuronic acid (G units) and poly- $\beta$-1,4-D-mannuronic acid ( $M$ units). The active site, carboxyl and hydroxyl groups on the

State Key Laboratory of Separation Membranes and Membrane Processes, School of Materials Science and Engineering, Tianjin Polytechnic University, Tianjin 300387, China

† Electronic supplementary information (ESI) available. See DOI: 10.1039/c8ra01324a backbone, can react with dye molecules, so alginate has high affinity and binding capacity to dye molecules. ${ }^{8}$ Especially, sodium alginate (SA) can be rapidly crosslinked with some metal ions in aqueous solution to form an gel network similar to an "egg-box", in which $\mathrm{Ca}^{2+}$ is considered as one of the satisfactory cross linking agent. ${ }^{9,10}$ Therefore, SA or the gel calcium alginate (CA) is recognized for their exceptional formability, ${ }^{\mathbf{1 1}}$ and can be used as a matrix material to immobilize ATP to prevent the secondary pollution in the process of sewage treatment.

Nevertheless, various studies are focused on individually adsorption or detection to some pollutant. The design of a novel material for simultaneous, quick and accurate detection and adsorption of pollutants, such as dye molecules, remains a challenge. Carbon dots (CDs) are a new type of nano-sized fluorescent materials which attract enormous attention due to the advantages of high fluorescence intensity and good photostability compared with conventional organic fluorochrome. Furthermore, CDs have merits of low molecular weight, small size, outstanding biological compatibility and low toxicity. ${ }^{\mathbf{1 2}}$ Extraordinarily, CDs play an important role in water quality monitoring due to the fluorescence and quenching properties. ${ }^{13,14}$ CDs can be used to detect metal ions, ${ }^{15}$ DNA, ${ }^{16}$ methylene blue $(\mathrm{MB})^{17}$ and so on ${ }^{18}$ because the fluorescence intensity will be changed when detecting the pollutants. However, CDs with fluorescence effect are often used in aqueous solution, which limited their applications, therefore, designing a solidstate fluorescent material is still a challenge. ${ }^{19}$ At present, CDs 
have been used in membrane and agarose materials via electrostatic interaction, while, they are easily dropped from the matrix materials.

In this work, ATP was immobilized by CA gel with excellent formability to improve the adsorption, and CDs were introduced into the surface of CA/ATP gel fibers through surface grafting method to obtain fluorescence performance, the fiberlike composites of CA/ATP- $g$-CDs gel fibers which had adsorption and fluorescence quenching abilities for MB simultaneous were prepared. The aim of "fiber-like" was to increase the specific surface area of the gel because high specific surface area would enhance the detection and adsorption.

\section{Experimental}

\section{Materials and reagents}

ATP was provided by Jiangsu Xuyuan halt non-metallic mining Ltd., which was treated with hydrochloric acid and dried in vacuum under $100{ }^{\circ} \mathrm{C}$ for $24 \mathrm{~h}$ before use, and the average diameter of ATP was 500 meshes. $^{20}$ SA was purchased from Sinopharm Chemical Reagent Co., Ltd. of Shanghai. Carbon dots (citric acid and ethylenediamine) were synthesized according to the reported procedure. ${ }^{21} \mathrm{KH}-560$ was obtained from Aladdin Industrial Co. All other chemicals used in this work were of analytical grade as received and deionized water was used to prepare all aqueous solutions.

\section{Preparation of CA/ATP- $g$-CDs gels}

Preparation of KH560-CDs. $50 \mathrm{mg}$ CDs were dispersed into $20 \mathrm{~mL}$ deionized water and then $2 \mathrm{~mL} \mathrm{KH-560} \mathrm{solution} \mathrm{were}$ added into CDs solution dropwise under continuously stirring at room temperature for $12 \mathrm{~h}$, and then the KH560-CDs were obtained. ${ }^{22}$ The modified reaction of CDs were illustrated according to the Scheme 1.

Preparation of CA/ATP gel fibers. $2 \mathrm{~g}$ SA was dissolved into $97.5 \mathrm{~g}$ deionized water to obtain the gel solution and then $0.5 \mathrm{~g}$ ATP was added slowly under continuously stirring at $25{ }^{\circ} \mathrm{C}$ until the mixed solution was well-dispersed. After vacuum defoaming, the mixed solution was transferred into a syringe and rapidly injected into $1000 \mathrm{~mL} 3 \mathrm{wt} \% \mathrm{CaCl}_{2} \cdot 2 \mathrm{H}_{2} \mathrm{O}$ solution where the CA/ATP gel fibers were formed and left overnight to allow complete gelation. Finally, CA/ATP gel fibers were thoroughly washed with deionized water to make them chloride free.

Preparation of CA/ATP-g-CDs gel fibers. CA/ATP gel fibers were immersed in KH560-CDs solution for $6 \mathrm{~h}$ under shaking condition and then thoroughly washed with deionized water to make them free from KH560-CDs. The preparation process of CA/ATP- $g$-CDs gel fibers and the surface grafting of CA/ATP gel fibers by KH560-CDs were illustrated in Scheme 2(a and b).

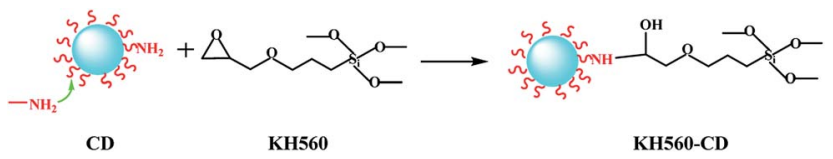

Scheme 1 Schematic illustration of the modification of CDs by $\mathrm{KH}$ 560.

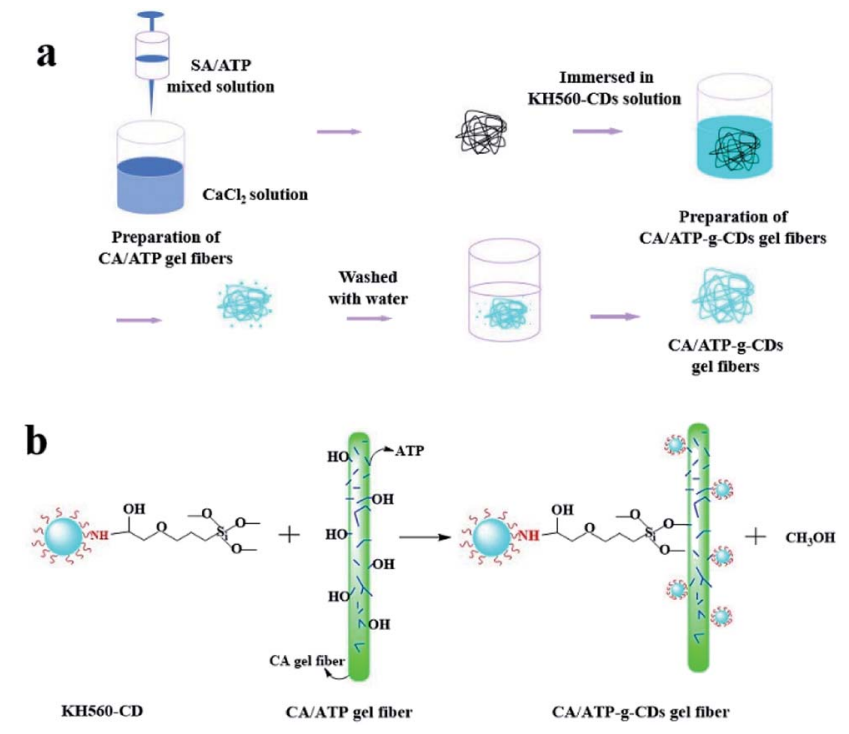

Scheme 2 Schematic of preparation of CA/ATP-g-CDs gel fibers and the surface grafting of CA/ATP gel fibers by KH560-CDs.

\section{Adsorption properties}

$10 \mathrm{mg}$ gel fibers adsorbent was added into $20 \mathrm{~mL} 20 \mathrm{mg} \mathrm{L}^{-1} \mathrm{MB}$ solution in a series of $50 \mathrm{~mL}$ conical flasks and then shocked in shaker at $25{ }^{\circ} \mathrm{C}$. The adsorption amount was calculated by the concentration of MB solution before and after adsorption which was obtained from the UV absorbance at $664 \mathrm{~nm}$ monitored by a UV-vis spectrometer (TU-1901, Japan).

\section{Fluorescence quenching properties}

$10 \mathrm{mg}$ CA/ATP- $g$-CDs gel fibers were transferred into a series of MB solutions $(10 \mathrm{~mL})$ with various concentrations for $6 \mathrm{~h}$, and then fluorescence spectra were monitored upon $446 \mathrm{~nm}$ excitation wavelength.

\section{Analysis and characterization}

The gel fibers were assessed morphologically using a Hitachi S4800 FE-SEM. AUTOSORBIQ of Quantachrome company was used for the specific surface area analysis. Thermogravimetric analysis was performed using a NETZSCH TGA (STA409PC), operated at nitrogen atmosphere from $25{ }^{\circ} \mathrm{C}$ to $900{ }^{\circ} \mathrm{C}$. X-ray photoelectron spectroscopy was accomplished using a Thermo Fisher Scientific (Waltham, MA, USA) K-Alpha spectrometer, operated at $50 \mathrm{eV}$ pass energy with a monochromatized $\mathrm{Al} \mathrm{K \alpha X-}$ ray source (1486.6 eV photons).

\section{Results and discussion}

In order to enhance the adsorption application of ATP in waste water, calcium alginate was selected as fixation materials to form CA/ATP hydrogel by the gelation of sodium alginate with $\mathrm{Ca}^{2+}$. The amounts of sodium alginate, $\mathrm{Ca}^{2+}$ and ATP ratio on the gelation process were investigated. A series of CA/ATP composite material was prepared (Fig S1†). Based on these 
data, the appropriate CA/ATP composite components can be preferably identified for the preparation CA/ATP gel.

\section{Characterization of CA/ATP- $g$-CDs gel fibers}

Fig. 1 showed the FE-SEM photographs of the gel fibers of pure CA and CA/ATP-g-CDs. The CA owned the fiber-like surface which corresponded to macroscopic gel fibers (Fig. 1c). The cross section of CA appeared some tiny bubbles which were caused by the air allowed into the fiber in the process of rapid injection (Fig. 1a). In spite of the tiny bubbles, the micrograph of pure CA was smooth while the micrograph of CA/ATP- $g$-CDs gel fibers was rough and wrinkled evidently and some pores emerged on the surface, indicating that the implanted ATP was conducive to improve the surface morphologies of CA.

ATP has a very large specific surface area for its fibrous morphology. Comparing to CA with the specific surface area of only $9.959 \mathrm{~m}^{2} \mathrm{~g}^{-1}$, the specific surface area of CA/ATP- $g$-CDs gel fibers were increased to $22.917 \mathrm{~m}^{2} \mathrm{~g}^{-1}$ by the introduction of ATP and CDs. ATP, as a fibrillar or rod-like single crystal, maybe acted as micro-channels in CA matrix and benefited for the improvement of specific surface area of the CA/ATP- $g$-CDs gel fibers ${ }^{23}$ which was correspond to the FE-SEM photographs.

To explore the content of ATP immobilized in CA, thermal stability was investigated by thermogravimetric analysis (TGA) under nitrogen atmosphere (Fig. 2). At 703. ${ }^{\circ} \mathrm{C}$, CA showed loss of weight while CA/ATP gel fibers had achieved a constant mass which was caused by the formation the looser structure through immobilizing ATP into CA. On the other hand, according to the final residue in turn to be calculated, the content of ATP immobilized into the CA was $22.4 \%$. Compared to the actual amount of ATP $(20 \mathrm{wt} \%(0.5 / 2.5))$ in the work, it can be verified that ATP was immobilized into CA ideally.

CDs can't attach to the surface of CA/ATP steadily due to the excellent water solubility. Therefore, CDs were grafted to the gel surface by the active group of $\mathrm{KH}-560$ to form stable fluorescence. As shown in Fig. 3a, a1 and a2 were samples of CA/ATP
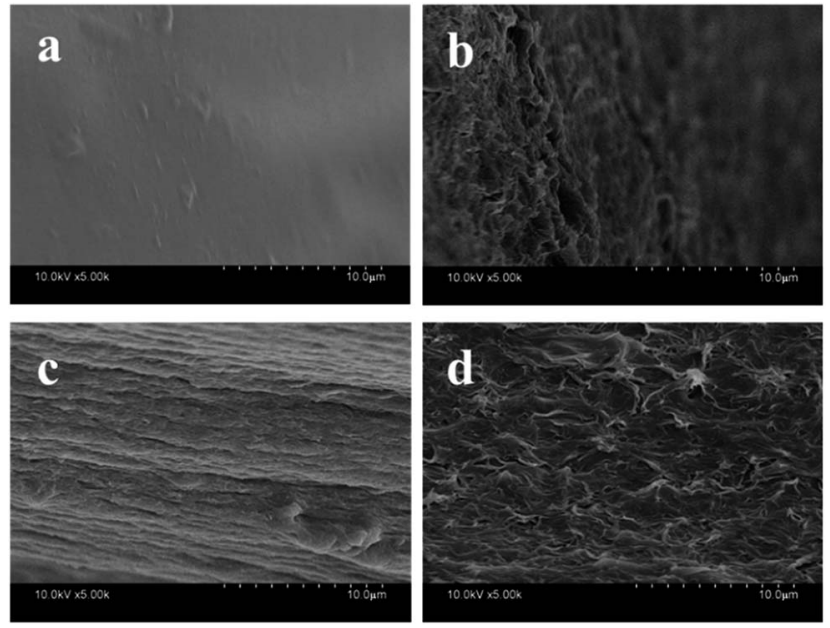

Fig. 1 SEM images of the cross-section ( $a$ and b), surface structure (c and $d$ ) of the prepared gel fibers: ( $a$ and $c)$ CA, (b and d) CA/ATP-gCDs.

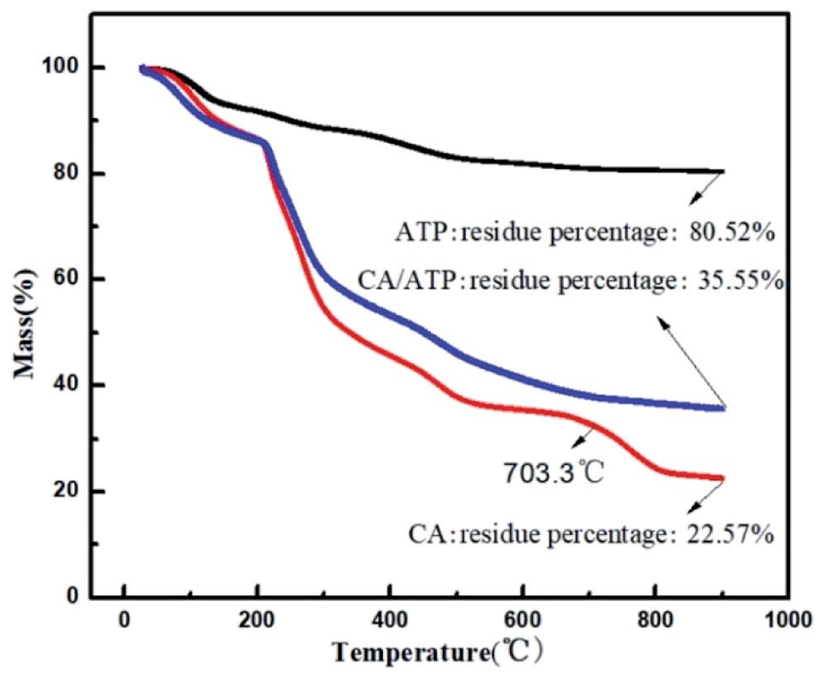

Fig. 2 Thermogravimetric analysis (TGA) curve of ATP, CA and CA/ATP gel fibers.

gel fibers immersed in CDs and KH560-CDs solutions respectively. Sample a2 appeared higher fluorescence intensity because CDs were easily dropped from the CA/ATP gel fibers in the process of water washing process while KH560-CDs can be grafted onto the CA/ATP gel fibers to form a stable chemical
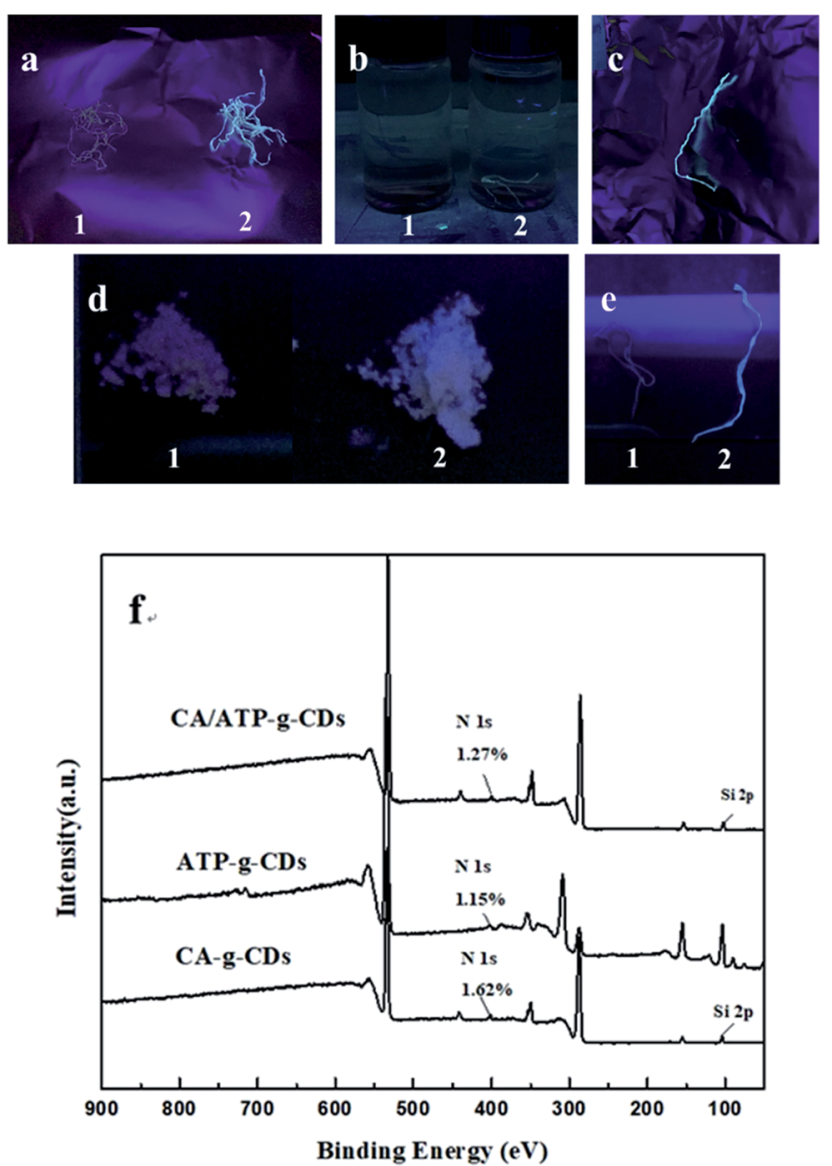

Fig. 3 Fluorescent images and XPS survey scan of CA-g-CDs and ATP-g-CDs. 
bond. In order to further verify the fluorescent stability in aqueous solution, the sample a1 and a 2 were immersed into pure water for 3 days, respectively, as b1 and b2 (Fig. 3b). Three days later, we found that after taking out the sample b2 from water, it still remained a strong blue light under UV radiation (Fig. 3c).

The $\mathrm{SiOCH}_{3}$ groups in $\mathrm{KH} 560-\mathrm{CDs}$ can react with hydroxyl groups in CA or ATP. To explore which kind of hydroxyl can reacted with KH560-CDs, CA- $g$-CDs and ATP- $g$-CDs materials were prepared by immersing pure CA gel fibers and ATP into KH560-CDs solution respectively. As shown in Fig. 3d and e, ATP- $g$-CDs (d2) and CA- $g$-CDs (e2) owned obvious fluorescence compared with pure ATP (d1) and CA (e1). XPS analysis was adopted to further confirm the reaction of CA/ATP gel fibers with KH560-CDs. The surface components of pure CA gel fibers and ATP had no amino-groups and pure CA gel fibers had no silicon oxygen bond either. But the XPS spectra showed two peaks at $402 \mathrm{eV}$ and $104 \mathrm{eV}$, which were attributed to $\mathrm{N} 1 \mathrm{~s}$ and $\mathrm{Si}$ $2 \mathrm{p}$ respectively. All of these indicated that KH560-CDs can react with the hydroxyl groups in both CA and ATP.

\section{Adsorption of the MB}

Gels have swelling properties and the excessive swelling is less conducive to water treatment for the taking of a large of water at the same time of adsorption by gels. As shown in Fig. 4, the swelling behaviors of CA/ATP- $g$-CDs gel fibers were surveyed and the swelling ratio was calculated according to the following equation:

$$
\mathrm{SR}=\left(m_{1}-m_{0}\right) / m_{0}
$$

where $m_{0}$ and $m_{1}$ respectively represent the weight of the gel fibers before and after swelling. The result showed that swelling process reached equilibrium quickly in $15 \mathrm{~min}$ and swelling ratio was as low as $1.1 \mathrm{~g} \mathrm{~g}^{-1}$. So it can be deduced that the swelling behaviors of CA/ATP- $g$-CDs gel fibers almost have no effect on the adsorption capacity.

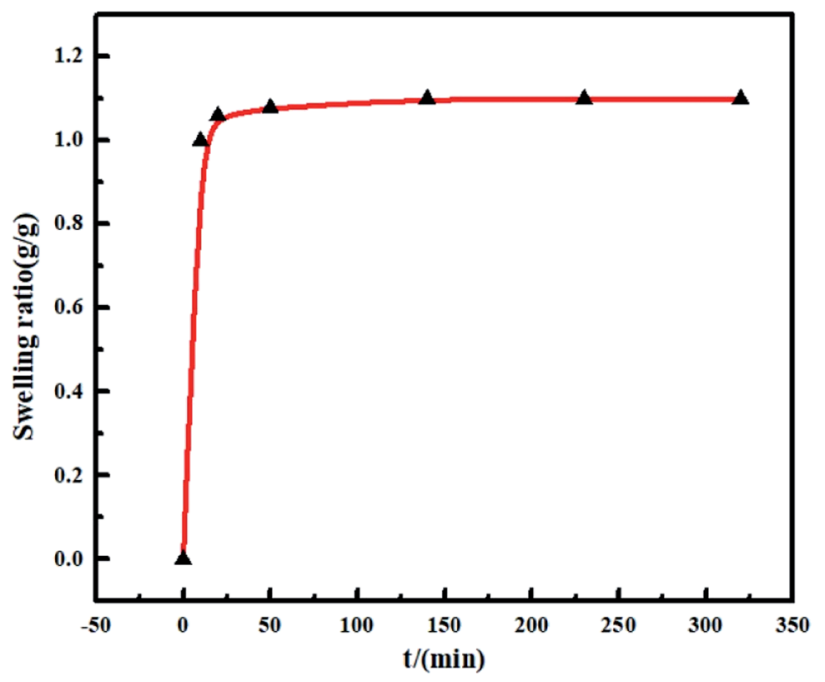

Fig. 4 Swelling ratio of CA/ATP-g-CDs gel fibers.

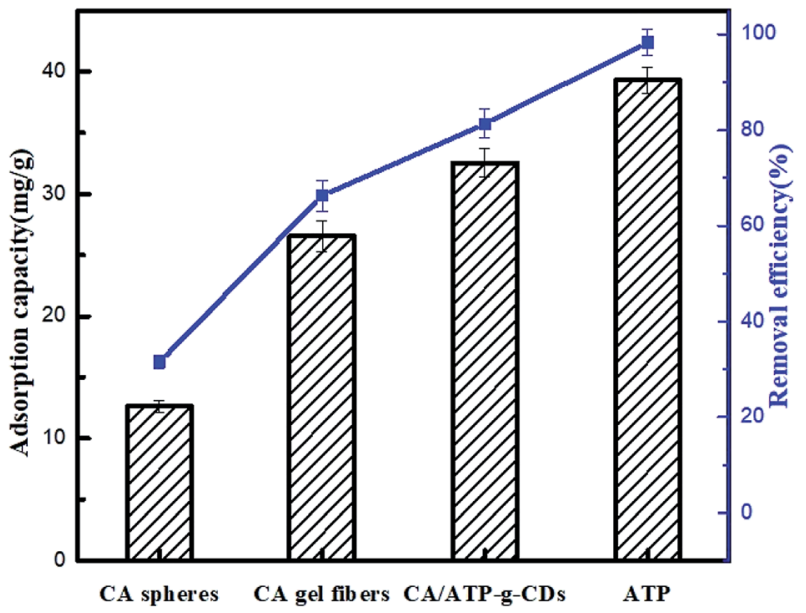

Fig. 5 MB adsorption of CA spheres, CA gel fibers, CA/ATP-g-CDs and ATP.

The adsorption of MB on different materials was shown in Fig. 5 and the results indicated that ATP had the very prominent adsorption capacity. Comparing with CA spheres, the adsorption capacity of CA gel fibers was improved because of the increase of the specific surface indicating that "fiber-like" gel had an advantage of higher adsorption capacity which was the reason why gel fibers were made in this work. CA/ATP- $g$-CDs gel fibers presented higher adsorption ability than pure CA because ATP was added into CA to produce many micro-channels thus the CA/ATP- $g$-CDs gel fibers owned higher specific surface area which could provide more adsorption sites.

The adsorption of MB onto CA/ATP- $g$-CDs gel fibers at different $\mathrm{pH}$ values was shown in Fig. 6 and the results clearly indicated that the adsorption capacity of CA and CA/ATP- $g$-CDs gel fibers for $\mathrm{MB}$ increased with the increase of $\mathrm{pH}$ value. In general, the adsorption of MB onto CA is very dependent on the $\mathrm{pH}$ value. At high $\mathrm{pH}$ values, the high adsorption ability was mainly attributed to the electrostatic interaction between positively charged MB and negatively charged carboxyl group (CA) or hydroxyl group (ATP). At low $\mathrm{pH}$ values, there were many $\mathrm{H}^{+}$in

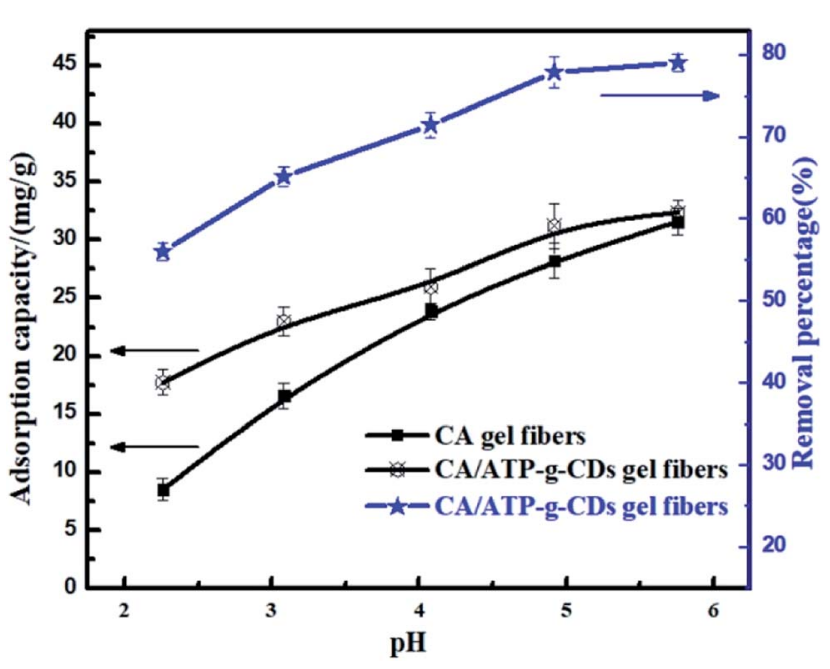

Fig. 6 Effect of $\mathrm{pH}$ of $\mathrm{MB}$ solution on adsorption. 
solution which competed with MB to combine with the limited active sites and so the electrostatic interaction between $\mathrm{MB}$ and CA/ATP-g-CDs gel fibers was weakened. So the adsorption capacity decreased. ${ }^{\mathbf{2}}$

\section{Fluorescence properties of the MB detection procedure}

In this paper, to systematically study the feasibility of the fluorescence detection of MB by CA/ATP- $g$-CDs gel fibers, a series of

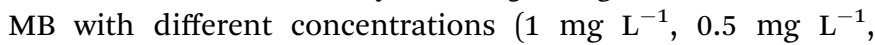
$0.1 \mathrm{mg} \mathrm{L}^{-1}$, and $1 \mu \mathrm{g} \mathrm{L}^{-1}$ ) was investigated (as shown in Fig. 7). The emission wavelength of the system was blue shifted right after immersing into $\mathrm{MB}$ solution which indicated the successful binding between MB and CA/ATP- $g$-CDs gel fibers. The intensity of emission spectra was gradually decreased with the increasing concentration of $\mathrm{MB}$ for that more $\mathrm{MB}$ molecules combined with fluorescence CDs, leading to the quenching of fluorescence. Energy change in emission spectrum $(\Delta E)$ of gel fibers was calculated according to the following equation:

$$
\Delta E=E_{1}-E_{2}
$$

where $E_{1}$ and $E_{2}$ represent the energy of emission peaks before and after binding with $\mathrm{MB}$. Therefore, the changes of emission peaks after binding with $\mathrm{MB}$ were plotted in Fig. 8. With the decreasing concentration of $\mathrm{MB}$, the energy change in emission peak decreased too. The CA/ATP- $g$-CDs gel fibers were found can detect MB down to a concentration of $1 \mu \mathrm{g} \mathrm{L} \mathrm{L}^{-1}$ or even lower in this work. Excitedly, the concentration of MB after detecting was as low as $70 \mu \mathrm{g} \mathrm{L}^{-1}$ or even lower (as shown in Fig. 8). This also corresponded to the above adsorbing experiments. Thus, it can conclude that the CA/ATP-g-CDs gel fibers can adsorb MB molecule. In this process, some of the MB combined with CDs on the gel fibers and produced fluorescence quenching by the acceleration of non-radiative electron/hole recombination through the electron transfer process, ${ }^{17}$ as shown in Scheme 3.

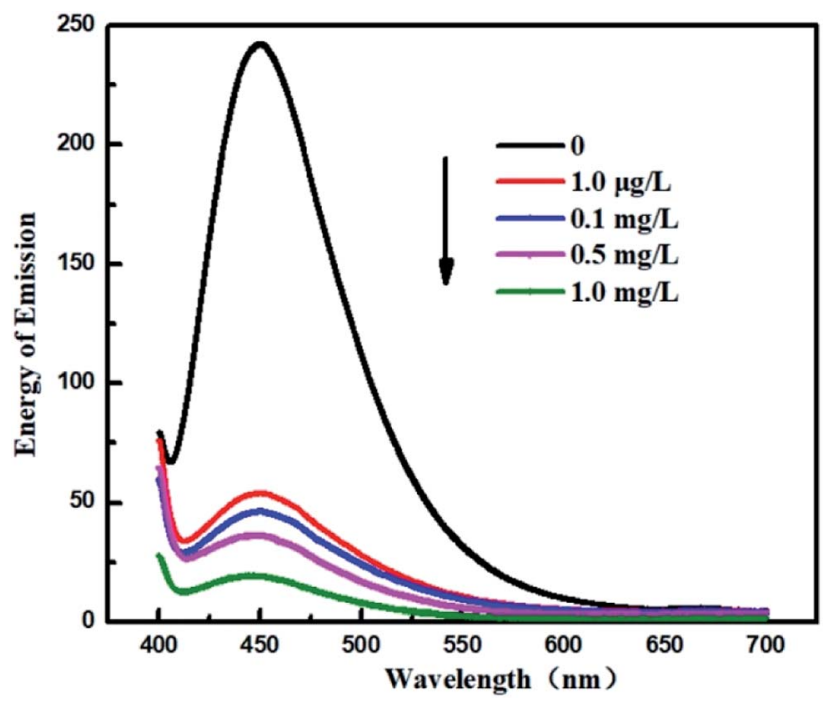

Fig. 7 Energy of emission before and after binding with MB solution with different concentration.

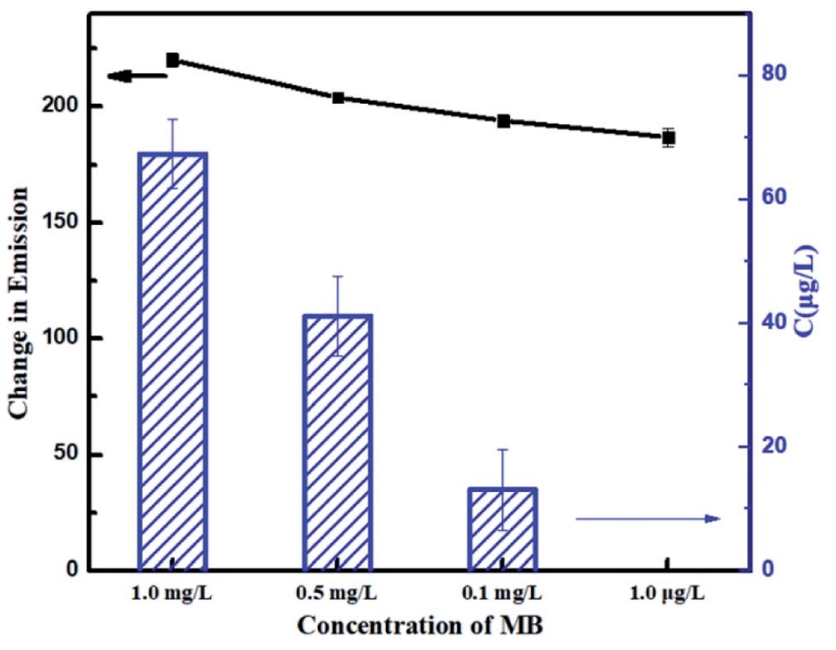

Fig. 8 Energy changes of emission peaks and MB concentration after detecting with different concentration of MB solutions.

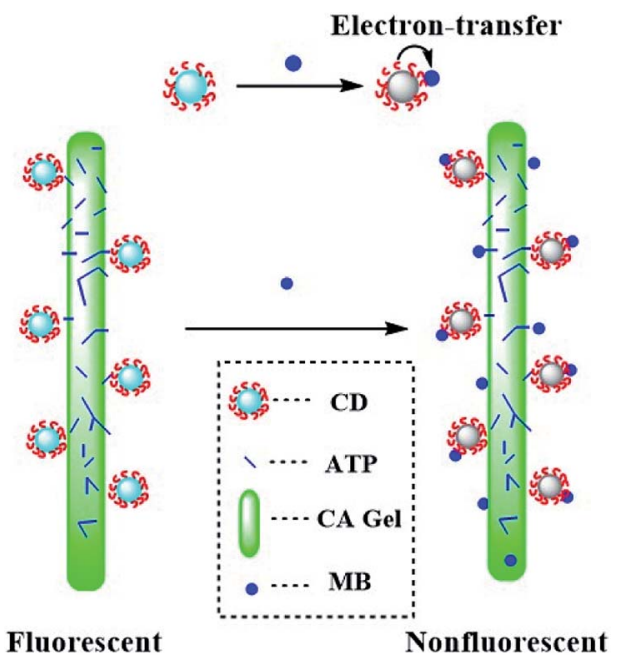

Scheme 3 Schematic illustration of quenching process of CA/ATP-gCDs gel fibers.

\section{Conclusions}

A novel fluorescence solid gel fibers CA/ATP- $g$-CDs had been successfully prepared for simultaneous detection and adsorption of MB. MB can be adsorbed by gel fibers while reducing the fluorescence of the CA/ATP- $g$-CDs. The satisfactory adsorption was derived from the increasing specific surface area of CA. The adsorption of CA/ATP- $g$-CDs gel fibers for MB was increased with the increase of $\mathrm{pH}$ value. The minimum detection limit was found to be as low as $1 \mu \mathrm{g} \mathrm{L}^{-1}$ through an electron-transfer process. At low concentration, the MB concentration became quite low and the removal efficiency could be as high as $100 \%$. These results showed that CA/ATP- $g$-CDs gel fibers were favorable materials to detect and adsorb MB simultaneously.

\section{Conflicts of interest}

There are no conflicts to declare. 


\section{Acknowledgements}

This work was supported by the National Natural Science Foundation of China (21174103, 21374078 and 51773152), the Natural Science Foundation of Tianjin (15JCYBJC17900) and the Program for Changjiang Scholars and Innovative Research Teams in University of Ministry of Education of China (IRT13084).

\section{Notes and references}

1 Y. Liu, Y. Kang, B. Mu and A. Wang, Chem. Eng. J., 2014, 237, 403-410.

2 L. Wang, J. Zhang and A. Wang, Desalination, 2011, 266(1), 33-39.

3 H. Cui, Y. Qian, Q. Li, Z. Wei and J. Zhai, Appl. Clay Sci., 2013, 72, 84-90.

4 H. Xu, J. Tang, L. Tan and W. Wang, Adsorpt. Sci. Technol., 2013, 31(6), 521-534.

5 Y. Deng, L. Wang, X. Hu, B. Liu, Z. Wei, S. Yang and C. Sun, Chem. Eng. J., 2012, 181, 300-306.

6 Y. Wang, W. Wang, X. Shi and A. Wang, Polym. Bull., 2013, 70(4), 1181-1193.

7 T. Wang, Y. Chen, J. Ma, Q. Qian, Z. Jin and Y. Zhang, Anal. Chem., 2016, 88(3), 1535-1541.

8 Y. Wang, W. Wang and A. Wang, Chem. Eng. J., 2013, 228, 132-139.

9 N. Gogoi and D. Chowdhury, J. Mater. Chem. B, 2014, 2(26), 4089-4099.
10 D. A. Rees and E. J. Welsh, Angew. Chem., Int. Ed. Engl., 1977, 16(4), 214-224.

11 D. L. Guerra, R. R. Viana, L. P. da Costa and C. Airoldi, J. Phys. Chem. Solids, 2009, 70(11), 1413-1421.

12 D. Chowdhury, N. Gogoi and G. Majumdar, RSC Adv., 2012, 2(32), 12156-12159.

13 Y. Fan, D. Shi, T. Zheng, K. Yun, X. Zhou and L. Chen, Sens. Actuators, B, 2016, 224, 926-935.

14 H. Li, Z. Kang, Y. Liu and S. T. Lee, J. Mater. Chem. B, 2012, 22(46), 24230-24253.

15 J. Liu, L. Lin, X. Wang, S. Lin, W. Cai, L. Zhang and Z. Zheng, Analyst, 2012, 137(11), 2637-2642.

16 H. Li, Y. Zhang, L. Wang, J. Tian and X. Sun, Chem. Commun., 2011, 47(3), 961-963.

17 W. Bai, H. Zheng, Y. Long, X. Mao, M. Gao and L. Zhang, Anal. Sci., 2011, 27(3), 243-246.

18 F. Yan, D. Kong, Y. Fu, Q. Ye, Y. Wang and L. Chen, J. Colloid Interface Sci., 2016, 466, 268-274.

19 N. Gogoi, M. Barooah, G. Majumdar and D. Chowdhury, ACS Appl. Mater. Interfaces, 2015, 7(5), 3058-3067.

20 G. Zhang, Y. Qin, C. Lv, X. Liu, Y. Zhao and L. Chen, Membrane Water Treatment, 2016, 7(3), 209-221.

21 S. Zhu, Q. Meng, L. Wang, J. Zhang, Y. Song, H. Jin and B. Yang, Angew. Chem., Int. Ed., 2013, 52(14), 3953-3957.

22 Y. Kim, H. Namgung, Y. Kim, G. Jang and T. S. Lee, Sci. Adv. Mater., 2014, 6(11), 2440-2444.

23 Y. Li, F. Liu, B. Xia, Q. Du, P. Zhang, D. Wang and Y. Xia, J. Hazard. Mater., 2010, 177(1), 876-880. 